\title{
EL PREVERBIO INSEPARABLE ZER- Y SUS IMPLICACIONES LINGÜÍSTICO- PRAGMÁTICAS EN LA ENSEÑANZA DEL ALEMÁN COMO LENGUA EXTRANJERA. UN ESTUDIO COMPARATIVO
}

\author{
Rafael López-Campos Bodineau
}

Using the separable and inseparble preverbs constitutes a great problem in learning German, because of the many lexical values they offer. Anyway, we think that learning many of them can be possible with a relatively well arranged way, in virtue of the semantic value of the preverbs and the base-verbs. In this article we will tray to make a clasification of all this verbs, and in the same way, we will tray to analyze if this procedure can give useful results for teaching german.

\section{Planteamiento del problema, delimitaciones y objetivos.}

El estudio de los preverbios de cualquier lengua constituye una cuestión sumamente compleja, debido fundamentalmente a la diversidad de valores léxicos que presentan, así como a la gran cantidad de funciones que desempeñan en el ámbito de la composición o derivación en que son empleados. Así, en líneas generales, podemos afirmar que uno de sus cometidos básicos es modificar la estructura sintáctica y/o semántica, no sólo del "verbo base"1, sino también, en muchos casos, de la oración completa en que aparecen. ${ }^{2}$ Especialmente relevante es en este sentido el caso de la lengua alemana ${ }^{3}$, debido, sin duda alguna, al alto número de preverbios de los que dispone, así como a la naturaleza tan heterogénea que presentan.

Muchas y muy controvertibles son las distintas clasificaciones que a lo largo del último siglo han sido propuestas en torno a los diferentes preverbios alemanes. Nosotros, por

\footnotetext{
${ }^{1}$ En relación con la designación "verbo base", véase el tratamiento expuesto en I. Kühnhold, H. Wellmann, Deutsche Wortbildung, Typen und Tendenzen in der Gegenwartssprache, Schwann, Düsseldorf, 1973.

${ }^{2}$ Vid. I. Barz, "Zur Wortbildungsaktivität primärer Lexeme". Beiträge zur Erforschung der deutschen Sprache 7 , Leipzig, 1987, págs. 4-11.

${ }^{3}$ Véase igualmente el caso de los preverbios romances ex, infra, inter, etc. en formaciones como extraere, infravalorar, interponer, etc. Vid. N. Filiceva, "Zur semantischen Isofunktionalität von Wortverbindung und Kompositum im Deutschen”. Deutsch als Fremdsprache 29, Leipzig, 1992, págs. 84-89.
} 
establecer algún tipo de restricción, pasaremos por alto cualquier consideración de tipo sintáctico o semántico, y nos limitaremos a clasificarlos según queden o no morfológicamente identificados con algún otro tipo de palabra. Así, distinguiremos simplemente entre "prefijos inseparables" (be-, emp-, ent-, er-, ver-y zer-), y "partículas de composición" (véanse en este último caso todos aquellos preverbios con identificación sustantiva: rad o maß en radfahren, maßgeben, etc.; adjetiva: hoch o sicher en hochspringen, sicherstellen, etc.; adverbial: los o weg en losfahren, weggehen, etc.; verbal: liegen, kennen o stehen en liegenlassen, kennenlernen, stehenbleiben, etc.; preposicional: $a b$, an, auf, über, um, etc. en über'setzen, um'fahren, abkleben, anbringen, etc.). ${ }^{4}$

Partiendo de esta distinción queremos tomar el siguiente hecho innegable como punto de partida: la aplicación de algunos de estos preverbios, así como de otros muchos mecanismos de formación de palabras, puede ser llevada a cabo de un modo completamente improvisado por parte del emisor/receptor en el transcurso de un proceso comunicativo cualquiera, tal y como expone Zifonun, o bien, más recientemente, otros como Wilss, Roggenhofer, Rickheit, Fandrych, W. Fleischer / I. Barz, Harras, Matussek. ${ }^{5}$ Esto quiere decir que si bien algunas composiciones con preverbios se muestran completamente "lexicalizadas" en la lengua alemana, es decir con valores de significado constantes e inalterables en el ámbito de un corpus de formaciones "previamente establecido", otras, sin embargo, podrían ser realizadas -llegado el momento- de un modo completamente "espontáneo". En este último caso se trata de mecanismos de derivación o composición aplicables a un número ilimitado de nuevas formaciones ${ }^{6}$ en virtud de los rasgos de significado tanto del preverbio como del verbo base. Dada la complejidad de cada uno de estos procesos hemos optado tan sólo por el estudio de los denominados "prefijos inseparables" y, más concretamente, por el caso particular de zer-, por cuanto se trata del preverbio que más claramente pone de manifiesto lo que acabamos de exponer. Ello se debe, en primer lugar, a que el corpus de verbos con zer- en lengua alemana es

\footnotetext{
${ }^{4}$ Véanse en este sentido las clasificaciones propuestas en P. Kunsmann, Verbale Gefüge, Hueber, München, 1973 , R. Hinderling, "Konkurrenz und Opposition in der verbalen Wortbildung". Tendenzen verbaler Wortbildung in der deutschen Gegenwartssprache, Helmut Buske Verlag, Hamburgo, 1982, págs. 81-107, K.-R. Harnisch, "Doppelpartikelverben als Gegenstand der Wortbildungslehre und Richtungsadverbien als Präpositionen. Ein syntaktischer Versuch”. Ibid, págs. 107-134. Asimismo, en relación con una diferenciación entre los conceptos de "partícula" y "prefijo", vid. B. Colbert, "Zur Frage der Präfixbildungen". Deutsch als Fremdsprache 3, Leipzig, 1966, págs. 12-16.

${ }^{5}$ G. Zifonun, Zur Theorie der Wortbildung am Beispiel deutscher Präfixverben, Hueber, Munich, 1973, W. Wilss, "Schematheorie und Wortbildung". Deutsch als Fremdsprache 28, Leipzig, 1992, págs. 230-233, AsbachSchnitker / J. Roggenhofer (Hg.), Neuere Forschungen zur Wortbildung und Historiographie der Linguistik. Tübingen, M. Rickheit, Wortbildung. Grundlagen einer kognitiven Wortsemantik, Opladen, 1993, Ch. Fandrych, Wortart, Wortbildungsart und kommunikative Funktion. Tübingen, 1993, W. Fleischer / I. Barz, Wortbildung der deutschen Gegenwartssprache. Tübingen, 1992, G. Harras (Hg.), Die Ordnung der Wörter, Berlín / New York, 1995, M. Matussek, Wortneubildung im Text. Helmut Buske Verlag. Hamburg, 1994.

${ }^{6}$ Preverbios como ge- en gehören, gerinnen, gedenken, etc., o bien emp- en empfangen, empfinden, empfehren, pese a ser portadores también de una rasgo "básico" ([+ unión] para ge- y [+ recepción] para emp-), no aparecen en ningún caso como verbos fundamentales en el ámbito de derivaciones de tipo proléptico, ni -en consecuenciapueden llevar a cabo lo que conocemos como "nuevas formaciones". Hemos de distinguir, por tanto, entre formaciones de tipo dinámico (aquellas compuestas por preverbios como er-, ver-y zer- que pueden ser aplicados ad hoc por el emisor/receptor), y estático (en aquellos otros casos como ge- y emp- en los que no es posible determinar este tipo de formaciones).
} 
relativamente reducido frente al de otros preverbios de su misma naturaleza como ver-, emp, ent-, er-, etc., y, en segundo lugar, a que se trata de un prefijo bastante homogéneo, al menos en lo relativo a su valor léxico.

Por tanto, según todo lo expuesto, es nuestro objetivo delimitar los distintos grupos de verbos con los que zer- resulta productivo e igualmente, partiendo de un corpus previamente establecido, analizar de qué modo es posible esta "normalización" de su uso en el ámbito de alguna otra formación similar. Para ello lo mejor será tomar como apoyo la descripción semántica de la base verbal de cualquiera de estas formaciones, y a partir de ahí estudiar la compatibilidad de zer- con cualquier otro verbo similar no incluido en nuestro corpus. Dada a la dificultad que plantea al estudioso de la lengua alemana el correcto uso de los mecanismos de formación de palabras en general -fundamentalmente si su lengua materna es el castellano o cualquier otra lengua romance-, consideramos que un estudio de estas características puede tener una aplicación muy interesante dentro del marco de la enseñanza del alemán como lengua extranjera. La agrupación de las formaciones con zeratendiendo a sus rasgos de significado, así como la delimitación de los distintos verbos base con los que resulta productivo, son cuestiones que -ni que decir tiene- pueden agilizar y simplificar su asimilación y aprendizaje.

\section{Descripción semántica.}

El número global de verbos formados con este prefijo disponible en la actualidad es bastante amplio, si bien en nuestro caso queremos partir únicamente de los contemplados en los registros publicados por G. Haensch, H. Müller/G. Haensch, Th. Schoen/ T. Noell, R.J. Slaby/ R. Grossmann, K. H. Bieler, K. H., E. Fröhlich, G. Kempcke, R. Klappenbach/W. Steinitz, K. Meil/ E. Arndt, H. Schumacher, G. Wahrig y G. Wahrig/ H. Krämer/H. Zimmermann (ver apéndice). ${ }^{7}$ Partiendo de todos ellos, podemos destacar dos características fundamentales que distinguen el uso de zer- frente al de todos los demás preverbios: su homogeneidad semántica y la naturaleza proléptica de todas sus formaciones. En relación con lo primero, podemos afirmar que zer- aparece en la práctica totalidad de los casos como portador del valor léxico relativo a la acción "deteriorar", e igualmente como indicador de un modo de acción "resultativo". Esto implica que el significado definitivo de cualquiera de estas formaciones no sólo incluye el de la acción

\footnotetext{
${ }^{7}$ La selección de estas 138 formaciones resulta de una labor de contraste a partir de los registros incluidos en G. Haensch, Diccionario Alemán. Alemán-Español y Español-Alemán, Barcelona, 1982, H. Müller / G. Haensch, Langenscheidts Handwörterbuch Spanisch-Deutsch, Berlin, 1987. Th. Schoen / T. Noell, Diccionario moderno Langenscheidts de los idiomas alemán y español, Berlin, 1987. R. J. Slaby / R. Grossmann, Diccionario de las lenguas española y alemana, 2 vols., Barcelona, 1987/1988. K. H. Bieler, Deutsche Verben im Kontext, München, 1981. Duden, Das große Wörterbuch der deutschen Sprache in 6 Bänden, Mannheim, 1976-1981. E. Fröhlich, Deutsches Wörterbuch, Herrsching, 1991. G. Kempcke (ed.), Handwörterbuch der deutschen Gegenwartssprache in zwei Bänden, 2 vols., Berlin, 1984. R. Klappenbach / W. Steinitz (eds.), Wörterbuch der deutschen Gegenwartssprache in sechs Bänden, Berlin, 1964-1977. K. Meil / E. Arndt, ABC der starken Verben, München, 1973. K. Meil / E. Arndt, $A B C$ der schwachen Verben, München, 1975. H. Schumacher (ed.), Verben in Feldern. Valenzwörterbuch zur Syntax und Semantik deutscher Verben, Berlin-New York, 1989. G. Wahrig (ed.), DtvWörterbuch der deutschen Sprache, München, 1980. G. Wahrig / H. Krämer / H. Zimmermann, Brockhaus-Wahrig deutsches Wörterbuch in sechs Bänden, Wiesbaden, 1980-1984.
} 
descrita por el verbo base, sino también el carácter resultativo derivado de los rasgos de significado de dicho preverbio. Véase, de este modo, la especial aplicación que encuentra en el ámbito de cualquiera de las siguientes tres acciones fundamentales:

1.) Acción resultativa "deteriorar" a partir de la marca fundamental "división en partes". Es el caso de los intransitivos zerbrechen, zerreißen, etc., o bien de los transitivos zerbeißen, zerbrechen, zerschneiden, zersägen, zerhacken, zerhauen, zerspalten, zerreißen, zernagen, zertrennen, zerkratzen, zermahlen, zerreiben, zerquetschen, etc.

2.) Acción resultativa "deteriorar" a partir de la marca fundamental "explotar". Véanse los intransitivos zerspringen, zerplatzen, zebersten, etc., o bien el transitivo zersprengen.

3.) Acción resultativa "deteriorar" a partir de la marca fundamental "golpear". Es el caso de los intransitivos zerknicken, zerknacken, zerklirren, etc., o bien de los transitivos zerschlagen, zerstoßen, zerprügeln, zerknicken, zerklopfen, zerknacken, zerknüllen, zertreten, zertrampeln, zerstampfen, etc. ${ }^{8}$

En segundo lugar también cabe destacar la naturaleza proléptica de todas estas formaciones, lo cual resulta evidente a partir de cualquiera de los grupos que hemos establecido. Así, apreciamos que los verbos registrados en 1.) quedan formados sobre la base semántica del verbo "fundamental" zerstören, de tal modo que, partiendo de una oración como er hat die Zeitung zerschnitten, es posible constatar la simultaneidad de dos acciones diferentes, correspondientes al verbo base schneiden y al mencionado "fundamental" zerstören. Esto implica que la formación zer-schneiden quedaría concebida como alternativa a la estructura etwas durch Schneiden zerstören, o bien, considerando el valor "resultativo" del preverbio, a etwas schneiden und es dabei zerstören. Véase, del mismo modo, en relación con otros verbos de ese mismo grupo:

$$
\begin{aligned}
\text { etwas zerspalten } & <\text { etwas durch Spalten zerstören } \\
& <\text { etwas spalten und es dabei zerstören } \\
\text { etwas zerbrechen } & <\text { etwas durch Brechen zerstören } \\
& <\text { etwas brechen und es dabei zerstören } \\
\text { etwas zersägen } & <\text { etwas durch Sägen zerstören } \\
& <\text { etwas sägen und es dabei zerstören }
\end{aligned}
$$

\footnotetext{
${ }^{8} \mathrm{Al}$ margen dejamos otros verbos con zer, caracterizados por describir esa misma acción resultativa "deteriorar" a partir de otras marcas menos homogéneas. Es el caso de formaciones como zerarbeiten, zerdehnen, zerdreschen, zerknittern, zerknüllen, zerkochen, zerlesen, zerlösen, zerpfeifen, zerpflücken, zerpflügen, zerprügeln, zerquälen, zerschießen, zerschleißen, zerschmettern, zersingen, zerstieben, zerstrahlen, zerstreuen, zerwerfen, etc. Si bien las bases de cualquiera de estos verbos no pertenecen a los tres grupos fundamentales que hemos establecido, no obstante sí se trata de bases a partir de las cuales puede quedar descrita la acción resultativa propia de dichos grupos. Véase durch langes Kochen zerfallen > zerkochen, etwas durch Einwerfen zerstören > etwas zerwerfen, etc.
} 


$\begin{array}{ll}\text { etwas zerteilen } & <\text { etwas durch Teilen zerstören } \\ & <\text { etwas teilen und es dabei zerstören } \\ \text { etwas zerreißen } & <\text { etwas durch Reißen zerstören } \\ & <\text { etwas reißen und es dabei zerstören } \\ \text { etwas zerbrechen } & <\text { etwas durch Brechen zerstören } \\ & <\text { etwas brechen und es dabei zerstören }\end{array}$

El mismo planteamiento puede quedar aplicado los verbos incluidos en 2.) y 3.), en la medida en que cualquiera de ellos aparece como portador de dos acciones verbales bien distinguidas la una de la otra, según los valores léxicos de cada uno de los componentes que las integran. Véase así, en relación con 2.), etwas zersprengen < etwas sprengen und es dabei zerstören < etwas durch Sprengen zerstören, etwas zerspringt < etwas Zerbrechliches fällt und wird dabei zerstört, etc., e igualmente en relación con 3.), etwas zerschlagen < etwas schlagen und es dabei zerstören < etwas durch Schlagen zerstören, etwas zertreten < auf etwas treten und es dabei zerstören < etwas durch Zertreten zerstören, zertrampeln < auf etwas trampeln und es dabei zerstören < etwas durch Trampeln zerstören, etc.

Al margen de estos grupos, hemos de șeñalar la posibilidad de aplicar estas dos mismas características a aquellas otras formaciones en las que el preverbio zer- queda unido a una base sustantiva. Es el caso de etwas zerbomben, zerstückeln, zerteilen, zergliedern, zerstäuben, zertrümmern, zerlöchern, zerfasern, zerkrümeln, zersplittern, zerbeulen, zerblättern, zerfleischen, etc. La formación de cualquiera de estos verbos con zer- responde a un proceso de modificación ${ }^{9}$ similar al anteriormente mencionado, si bien el valor léxico de la base vendría dado en estos casos por los rasgos de significado propios del sustantivo a partir del cual es derivada. Así, según todo esto, y atendiendo a la relación que presente dicha base respecto del preverbio, podremos distinguir los siguientes grupos fundamentales:

4.1.) Formaciones en las que la base sustantiva establece el instrumento mediante el cual es llevada a cabo la acción "deteriorar": etwas mit Bomben zerstören > zerbomben, mit einer Beule beschädigen > zerbeulen.

4.2.) Formaciones en la que la base sustantiva designa aquello en lo que queda transformado el objeto sobre el cual es aplicada dicha acción "deteriorar": etwas in kleine Stücke schlagen und es dabei zerstören > zerstückeln, etwas in Stücke teilen und es dabei zerstören > zerteilen, etwas in Glieder teilen und es dabei zerstören $>$ zergliedern, etwas zu Trümmern machen und es dabei zerstören > zertrümmern,

\footnotetext{
${ }^{9}$ En relación con una concepción de los procesos de formación de palabras como resultado de un proceso transformacional, vid. J. Lerot, Transformationelle Behandlung der Verbzusammensetzungen im Deutschen, Schwann, Düsseldorf, 1971, G. Zifonun, Zur Theorie der Wortbildung am Beispiel deutscher Präfixverben, Hueber, Munich, 1973., R. Steinitz, "Mehrdeutigkeit in der Grammatik auf und an - eine Fallstudie". Fügungspotenzen, Akademie Verlag, Berlín, 1992, págs. 163-188, S. Sarlov, Zur semantischen Struktur von Präfixverben, Niemeyer, Tubinga, 1992, M. Schröder, "Warum nicht *umweltlich und *Umweltler?". Deutsch als Fremdsprache 30, Leipzig, 1993, págs. 172-175.
} 
y del mismo modo en relación con zerlöchern, zerfasern, zerkrümeln, zersplittern, zerkleinern zerfleischen, zerblättern, etc.

Especialmente relevante resulta el caso de zerkleinern -en el ámbito de un último grupo al que nos referiremos como 5.)-, ya que el valor léxico del verbo resultante queda determinado por los rasgos de significado de un adjetivo, en este caso klein (véase etwas in kleine Stücke zerteilen und es dabei zerstören > etwas zerkleinern).

Una vez establecidos los diferentes grupos léxicos con zer-, así como el carácter proléptico propio de todas sus formaciones, analicemos a continuación hasta qué punto podemos "normalizar" el uso de dicho preverbio en el ámbito de nuevos verbos. En lo que se refiere a las formaciones registradas en 1.), apreciamos que todas ellas describen el valor resultativo "deteriorar" sobre una acción verbal marcada por el rasgo fundamental [+ división en partes] (véase zer- + beißen, brechen, schneiden, sägen, hacken, hauen, spalten, reißen, nagen, trennen, mahlen, reiben, quetschen, etc.):

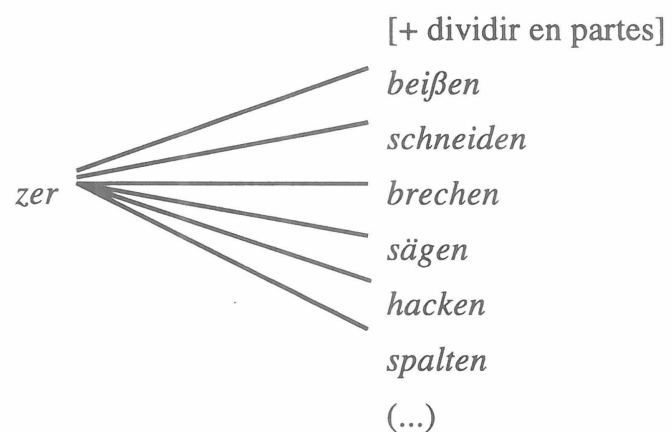

La productividad de zer con este tipo de verbos hace posible su compatibilidad con todos aquellos verbos base que sean portadores de ese mismo rasgo. Véase, por ejemplo, a partir de kratzen, kauen, etc., la posibilidad de obtener alguna otra formación similar como zerkauen, zerkratzen, etc. (etwas kauen > etwas zerkauen, etwas kratzen > etwas zerkratzen, etc.), tratándose en este caso de verbos con un uso completamente correcto y habitual. Todo ello nos llevaría a la expresión: ${ }^{10}$

$$
\begin{array}{lll}
\text { Compos. zer }+\mathrm{VB} & +> & \text { zer } \gg \text { [+ resultat: deteriorar] } \\
& +> & \mathrm{VB} \gg>\text { [+ dividir en partes }]
\end{array}
$$

Exactamente del mismo modo podríamos proceder en relación con los verbos incluidos en 2.) ó 3.), ya que partiendo de zerbersten, zerplatzen, zerspringen, etc. o bien de

\footnotetext{
${ }^{10}$ Vid. L. Kreiser, S. Gottwald, W. Stelzner (Eds.), Nichtklassische Logik, Berlin, Akademie Verlag, 1990, B. H. Partee, A. Meulen, R. E. Wall, Mathematical Methods in Linguistics, Dordrecht, Kluwer, 1990.
} 
zerschlagen, zerstoßen, zertrampeln, etc., respectivamente, podremos obtener alguna otra formación similar como zerknallen, zerdrücken, zerstechen, etc., cuya aplicación resulta igualmente adecuada, desde un punto de vista tanto gramatical como lingüísticopragmático. ${ }^{11}$ En resumidas cuentas, según todo lo expuesto, sería posible establecer:

\begin{tabular}{|l|c|c|l|l|}
\hline & Acción resultativa & $\begin{array}{c}\text { Marca } \\
\text { fundamental }\end{array}$ & \multicolumn{1}{|c|}{ Ejemplos } & $\begin{array}{c}\text { Formación de nuevos } \\
\text { verbos }\end{array}$ \\
\hline zer $_{1}$ & "deteriorar" & "dividir en partes" & $\begin{array}{l}\text { zerbrechen, } \\
\text { zerschneiden, } \\
\text { zersägen, etc. }\end{array}$ & $\begin{array}{l}>\text { zerkauen, } \\
>\text { zerkratzen, etc. }\end{array}$ \\
\hline zer $_{2}$ & "deteriorar" & "explotar" & $\begin{array}{l}\text { zerspringen, } \\
\text { zerplatzen, } \\
\text { zerbersten, etc. }\end{array}$ & $\begin{array}{l}>\text { zerknallen, } \\
\text { etc. }\end{array}$ \\
\hline zer $_{3}$ & "deteriorar" & "golpear" & $\begin{array}{l}\text { zerschlagen, } \\
\text { zerklirren, } \\
\text { zerknacken, } \\
\text { zertreten, } \\
\text { zertrampeln, } \\
\text { zerstampfen, } \\
\text { etc. }\end{array}$ & $>$ zerdrücken, \\
\hline
\end{tabular}

Ahora bien, el hecho de que esto resulte evidente en el ámbito de estos tres grupos principales no implica que haya de ser así en todos los demás casos. Véase así la imposibilidad de establecer un planteamiento similar en aquellas otras formaciones en las que zer- queda unido a una base sustantiva. Así, en lo que se refiere a 4.1.) apreciamos que pese a ser posible establecer derivaciones de este tipo a partir de algún sustantivo como Bombe, no por ello será posible proceder del mismo modo en el marco de otros similares como Kugel, Pfeil, etc. (compárese etwas mit einer Bombe zerstören > etwas zerbomben con etwas mit einer Kugel zerstören > *etwas zerkugeln o etwas mit einem Pfeil zerstören $>$ *etwas zerpfeilen).

Exactamente el mismo planteamiento puede quedar aplicado a los verbos incluidos en 4.2.), pues a partir de formaciones como etwas zerstückeln, zerstäuben, zertrümmern, zerpulvern, etc. resultará imposible la formación de otras como *zerbreien, etc. (compárese

\footnotetext{
${ }^{11}$ En relación con este último grupo cabe destacar el caso de los verbos base knicken, knacken, klirren, klopfen, knüllen y algunos otros más, los cuales comparten la peculiaridad de ser onomatopéyicos (véase así die Hunde haben die Stücke Knochen zerknackt, wir haben alle Zweige zerknickt, er hat alles Mögliche mit einem Hammer zerklopft, etc., o bien er der ganze Baum zerknickte unter dem Gewicht des Hauses). Si todos ellos resultan compatibles con zer-, es importante aclarar que ello es debido únicamente al valor léxico "impactar" del que son portadores y no a la motivación fonética con la que se les vincula. Esto queda de manifiesto a partir de la imposibilidad de determinar formaciones con zer- en el ámbito de cualquier otro verbo similar no referido a tal acción "impactar" (knistern > *zerknistern, knarren > *zerknarren, knurren > *zerknurren, etc.).
} 
etwas zu Stückeln machen > etwas zestückeln, etwas zu Trümmern machen > etwas zertrümmern, etc. con etwas zu Brei machen $>$ * etwas zerbreien, etc.), pese a ser las bases $B r e i$, etc. portadoras de los rasgos léxicos fundamentales compatibles con dicho preverbio. Algo parecido ocurre también en relación con el único verbo recogido con base adjetiva, en este caso zerkleinern, ya que si bien se trata de un verbo plenamente "lexicalizado" en la lengua alemana actual, sin embargo no por ello será posible tomar alguna otra base adjetiva similar como winzig, eng, etc. para la obtención de nuevas formaciones. Compárese así etwas zu kleinen Teilen od. Stücken machen und es dabei zerstören > etwas zerkleinern frente a etwas zu ganz winzigen Teilen od. Stücken machen und es dabei zerstören > *etwas zerwinz(ig)en, etc. Así, en este caso, podríamos establecer: ${ }^{12}$

\begin{tabular}{|c|c|c|c|c|}
\hline & Acción resultativa & $\begin{array}{c}\text { Marca } \\
\text { fundamental }\end{array}$ & Ejemplos & $\begin{array}{c}\text { Formación de nuevos } \\
\text { verbos }\end{array}$ \\
\hline$z e r_{4.1}$ & "deteriorar" & "dividir en partes" & zerbomben, etc. & $\begin{array}{l}>*_{\text {zerkugeln, }} \\
>*_{\text {zerpfeilen, etc. }}\end{array}$ \\
\hline$z^{2 e r_{4.2}}$ & "deteriorar" & "dividir en partes" & $\begin{array}{l}\text { zertrümmern, } \\
\text { zerpulvern, } \\
\text { zerstückeln, etc. }\end{array}$ & $\begin{array}{c}>{ }^{*} \text { zerbreien, } \\
\text { etc. }\end{array}$ \\
\hline$z e r_{5}$ & "deteriorar" & "dividir en partes" & zerkleinern & $\begin{array}{l}>{ }^{*} \text { zerwinz(ig)en, } \\
\text { etc. }\end{array}$ \\
\hline
\end{tabular}

A la vista de todo lo expuesto resulta asimismo especialmente relevante el caso de formaciones intransitivas como zergehen, zerfahren, zerlaufen, zerrinnen, zerfließen, zerschmelzen, zerfallen, etc., o también de las transitivas zerlegen, zersetzen, zerlassen, etc., es decir de todas aquellas uniones con zer- en las que el verbo base designa un "desplazamiento". En todas ellas encontramos igualmente la aparición de estructuras en cierta medida prolépticas (véase etwas zerschmilzt < etwas schmilzt und wird dabei zerstört, etc.), sin que por ello -a diferencia de los anteriores grupos establecidos- podamos determinar la existencia criterios generales que rijan la productividad de las mismas. De este modo, pese a ser posible la formación de los intransitivos zerlaufen, zergehen,

\footnotetext{
${ }^{12}$ En lo que se refiere a la posibilidad de obtener nuevas formaciones en el ámbito de cualquiera de estos grupos, hemos de hacer algunas salvedades, por ejemplo la relativa a todos aquellos verbos de origen latino que fueron incorporados a la lengua alemana mediante la aplicación del sufijo -ieren. Véase así, en relación con los registrados en 2.), la necesidad de excluir de este planteamiento a explodieren, detonieren, hochgehen, etc., pese a designar todos ellos igualmente la acción "explotar", propia de los verbos base registrados en dicho grupo: *zerexplodieren, *zerdetonieren, *zerhochgehen, etc. Del mismo modo también hemos de destacar la imposibilidad de obtener verbos con zer- en aquellos otros casos en los que el verbo base ya aparece como resultado de la aplicación de cualquier otro mecanismo de prefijación. Véase en este sentido que si bien es posible una formación con zer- a partir de los verbos base prügeln o teilen, por ser ambos portadores de los rasgos necesarios para ello -tal y como ha quedado expuesto-, sin embargo no podríamos afirmar lo mismo en el caso de verprügeln o aufteilen, pese a quedar dichos verbos marcados con una dotación similar. (compárese prügeln > zerprügeln, teilen $>$ zerteilen frente a verprügeln $>$ *zerverprügeln, aufteilen $>$ *zeraufteilen, etc.).
} 
zerfallen, etc., en las cuales las bases quedan ocupadas por verbos de desplazamiento (véanse en este sentido oraciones como die Schokolade ist zerlaufen, das Haus ist zerfallen, etc.), no por ello resultará factible la construcción de otras similares como *zersinken, *zersenken o *zerfliegen. Todo esto implica que la acción resultativa "destruir" habrá de ser aplicada a estos últimos verbos, no mediante una formación con zer-, sino mediante el empleo de alguna otra estructura alternativa $(*$ das Schiff ist zersunken $>$ das Schiff ist gesunken und dabei zerstört worden, *das Flugzeug ist zerflogen > das Flugzeug ist abgestürzt und dabei zerstört worden). Véase en este mismo sentido la imposibilidad de obtener composiciones como *zerstellen, *zerhängen, etc. pese a ser practicable la unión de zer- con alguno de los transitivos pertenecientes a este mismo grupo (zerlegen, etc.).

En lo que se refiere a estas ultimas formaciones mencionadas, hemos de destacar también la imposibilidad de establecer cualquier tipo de pluralización de tipo lingüísticopragmático, pues el carácter gramatical de las mismas estará en gran medida condicionado por el valor léxico de los restantes elementos oracionales, fundamentalmente del actante sujeto. Véase así la clara aplicación que muestra el verbo zerfallen tan sólo en aquellos casos en que es descrito algún tipo de "desgaste", "corrosión" o "consunción". Compárese die Mauern sind runtergefallen und dabei zerstört worden > die Mauern sind zerfallen, etc. con das Glas ist mir runtergefallen und (ist) dabei zerbrochen $>$ *das Glas ist mir zerfallen, etc.

Todo esto tiene un especial interés si es analizado desde un punto de vista comparativo. Así, apreciamos que las lenguas romances en general, como por ejemplo el castellano, carecen de estructuras prolépticas en torno a las marcas semánticas representadas por el preverbio alemán zer-, por lo que cualquier formación de verbos como zerschneiden, zerbrechen, zersägen, etc. habrá de ser traducida o interpretada mediante el empleo de otras construcciones alternativas, normalmente más complejas. En este sentido, lo más importante a destacar, es la importancia tanto del preverbio como del verbo base en el proceso de formación, de tal modo que cualquier traducción de estos verbos en la que se prescinda del valor léxico de alguno de dichos componentes, podría dar como resultado una versión no del todo correcta. Véase así, partiendo de la oración er hat alles zerschnitten, que traducciones como lo ha recortado todo o lo ha destrozado todo no podrían resultar en ningún caso válidas, en la medida en que en ellas tan sólo son contemplados los rasgos de significado propios de la base schneiden o bien del preverbio zer-, respectivamente. Por ello, una buena traducción del verbo zerschneiden -así como de cualquier otro formado con dicho preverbio- ha de tener en consideración los rasgos de significado de los dos componentes de la formación, por lo que la oración antes indicada tan sólo podría ser formulada como lo ha destrozado al recortarlo o bien lo ha recortado hasta dejarlo completamente deteriorado. ${ }^{13}$

\footnotetext{
${ }^{13}$ En lo que se refiere a todo esto consideramos especialmente relevante la contemplación de los rasgos de significado del componente zer-, por cuanto queda en oposición a otros preverbios alternativos como $a b$, entzwei, durch, etc., como se aprecia a partir de la oposición existente entre zerschneiden, abschneiden, entzweischneiden, durchschneiden, etc. Todo esto nos lleva a pensar en la importancia del valor léxico del preverbio, ya que se trata de un componente oracional portador de una carga semántica elemental para la determinación del modo de acción concreto en que ha de ser situado el proceso descrito por el verbo base, en este caso schneiden.
} 
Exactamente lo mismo podemos aplicar a los verbos incluidos en 2.) y 3.), en la medida en que también en estos casos es necesario recurrir a construcciones más complejas, a la hora de buscar una buena traducción. Véase así, en lo que se refiere a las formaciones comprendidas en 2.), la necesidad de interpretar verbos como zerspringen, zerknallen, zerplatzen o zerberrsten, no simplemente como explotar, destrozar, destruir, o alguna acción similar, sino más bien como explotar hasta quedar completamente destrozado, o bien destrozar mediante una explosión, o, como consecuencia de una explosión. Algo parecido ocurre en relación con los verbos englobados en 3.), ya que tampoco en estos casos bastará con traducir verbos como zerschlagen, zertrampeln, zerdrücken, etc. como golpear, pisotear u oprimir, respectivamente, sino que será necesaria una versión más compleja -difícilmente abarcable en castellano mediante un único verbo- como golpear, pisotear u oprimir hasta quedar completamente destrozado, o bien destrozar algo golpeándolo, pisoteándolo u oprimiéndolo.

Un planteamiento similar puede quedar atribuido a la mayor parte de las formaciones incluidas en el resto de los grupos anteriormente mencionados, siendo en este sentido especialmente relevante la posibilidad de abordar de un modo comparativo el estudio de los criterios de productividad en torno a dicho preverbio. Véase en este sentido, en lo que se refiere por ejemplo a los verbos incluidos en 1.), la correspondencia existente entre zer-y el uso en castellano de cualquier otro verbo mediante el cual quede descrita la ya mencionada acción resultativa "deteriorar" a partir de la marca fundamental "división en partes":

$\begin{array}{lll}\text { romper algo hasta quedar destrozado } & = & \text { etwas zerbrechen } \\ \text { serrar algo hasta quedar destrozado } & = & \text { etwas zersägen } \\ \text { cortar algo hasta quedar destrozado } & = & \text { etwas zerschneiden } \\ \text { roer algo hasta quedar destrozado } & = & \text { etwas zernagen, }\end{array}$

etc.

Así, partiendo de estas identificaciones, también se podrá estar en condiciones de formar "nuevos verbos" alemanes con zer. Para ello bastará con partir de expresiones similares a las que acabamos de exponer (p. ej. masticar, arañar, rasgar, moler algo hasta dejarlo completamente deshecho o maltrecho) y aplicar el preverbio a las bases verbales correspondientes a cada una de dichas acciones -en este caso kauen, kratzen, beißen o mahlen-. Véase:

$\begin{array}{lll}\text { masticar alg. hasta dejarlo deshecho } & = & \text { etwas zerkauen } \\ \text { arañar alg. hasta dejarlo compl. maltrech } & = & \text { etwas zerkratzen } \\ \text { rasgar algo hasta destrozarlo } & = & \text { etwas zerreißen } \\ \text { moler algo compl., hasta dejarlo deshecho } & = & \text { etwas zermahlen, }\end{array}$

etc.

Exactamente el mismo planteamiento puede quedar aplicado a los verbos incluidos en 2.) y 3.). Véase zerknallen, zerbersten, etc. > estallar/explotar hasta quedar destrozado, 
zerschlagen > golpear hasta quedar destrozado, zertreten > pisar hasta quedar destrozado, etc.

Los dos aspectos fundamentales que han quedado expuestos pueden ser, por tanto, fácilmente aprovechables para la didáctica del alemán como lengua extranjera, sobre todo en lo que se refiere al desarrollo de la competencia oral. Así, en primer lugar, y como ya ha quedado expuesto, pensamos que la capacidad del estudiante para formar "nuevos verbos" con zer puede ser ampliamente desarrollada en el ámbito de una unidad didáctica con grandes posibilidades de éxito, si bien ello requiere por supuesto un amplio conocimiento por parte del docente de las formaciones de partida "ya existentes" -dicho de otro modo, de aquellas que aparecen como "lexicalizadas"-, así como una absoluta seguridad en torno la corrección gramatical de los verbos de nueva creación, cuyo uso se intenta "estimular". Es decir, en relación con esto último, el docente habrá de saber en todo momento qué "nuevas formaciones" son practicables y qué otras suponen una infracción gramatical en torno al uso de dicho preverbio. Esta labor de corroboración se derivará de su propia experiencia lingüística -si se trata de un profesor nativo-, o bien de la previa selección de un corpus al que se llegará mediante una minuciosa tarea de contraste y confrontación con el uso real de la lengua a impartir, en este caso la alemana. Ello evitará la formación de estructuras gramaticalmente incorrectas como las anteriormente mencionadas *mir ist das Glas zerfallen, etc., o bien la creación de verbos como *zerstellen, *zerhängen, *zerbreien, etc.

Asimismo, también queremos llamar la atención sobre la importancia de abordar de un modo comparativo la docencia de los verbos con zer -así como en relación con otros muchos procesos de formación de palabras-, fundamentalmente si ésta queda orientada a estudiantes universitarios hispanohablantes, pues tan sólo desde un planteamiento de estas características será posible recalcar el tipo de acción resultativa a la que aluden todos ellos, así como el componente léxico completo del que son portadores.

\section{Conclusiones.}

A la vista de todo lo expuesto podemos establecer las siguientes conclusiones fundamentales:

1. El preverbio zer- presenta en todas sus formaciones un único tipo de acción resultativa, así como un valor léxico constante. No obstante, en virtud de los rasgos semánticos de los verbos con los que resulta compatible, podemos establecer tres grupos "fundamentales" de formaciones correspondientes a las acciones "dividir en partes", "explotar" y "golpear". A estos grupos hemos de añadir los numerosos casos en que zer- aparece en unión a verbos con base sustantiva, o bien, en algún caso, adjetiva.

2. El preverbio zer-puede ser ampliamente aplicado a partir de los tres grupos de verbos "fundamentales", siempre y cuando sean respetados los rasgos de significado básicos de sus dos componentes principales. Este planteamiento no puede ser constatado en el ámbito de las formaciones de zer- en unión a verbos con base sustantiva, o bien en aquellos otros casos en que la base verbal designa un "estado" o "desplazamiento". 
3. Las formaciones con zer- muestran una naturaleza de tipo proléptico, si las comparamos con sus equivalencias en otras lenguas, siendo especialmente digno de destacar el caso de las romances. De cualquier modo, ello no evita que los mecanismos alternativos a dicho preverbio en cualquiera de estas otras lenguas muestren una misma homogeneidad similar.

4. La problemática planteada en torno a la posibilidad de obtener nuevas formaciones con zer- también puede ser abordada desde un punto de vista puramente comparativo -véanse en este sentido el caso de las formaciones a partir de los verbos incluidos en 1.), 2.) y 3.)-, lo cual habrá de ser llevado a cabo, naturalmente, según los procedimientos lexicológicos propios de cada una de las lenguas que entren en consideración.

5. Todo esto es también aplicable a la enseñanza del alemán como lengua extranjera, en la medida en que la correcta aplicación de cualquiera de estos procedimientos podrá hacer -sin duda alguna- relativamente ágil y eficaz el estudio y asimilación de este tipo de formaciones. 


\section{Apéndice}

Indicamos a continuación la totalidad de verbos con zer- incluidos en los registros anteriormente citados, y que hemos tomado como punto de partida:

$\begin{array}{llll}\text { zerarbeiten } & \text { zerbeißen } & \text { zerbersten } & \text { zerbeulen } \\ \text { zerblättern } & \text { zerbomben } & \text { zerbrechen } & \text { zerbröckeln } \\ \text { zerbröseln } & \text { zerdehnen } & \text { zerdeppern } & \text { zerdreschen } \\ \text { zerzausen } & \text { zereisen } & \text { zerfahren } & \text { zerfallen } \\ \text { zerfasern } & \text { zerfetzen } & \text { zerflattern } & \text { zerfledern } \\ \text { zerfleischen } & \text { zerfließen } & \text { zerfransen } & \text { zerfressen } \\ \text { zerfurchen } & \text { zergehen } & \text { zergliedern } & \text { zergrübeln } \\ \text { zerhacken } & \text { zerhäckseln } & \text { zerhauen } & \text { zerkauen } \\ \text { zerkleinern } & \text { zerklirren } & \text { zerklopfen } & \text { zerklüften } \\ \text { zerknacken } & \text { zerzupfen } & \text { zerknäulen } & \text { zerknicken } \\ \text { zerknirschen } & \text { zerknittern } & \text { zerknüllen } & \text { zerkochen } \\ \text { zerkörnen } & \text { zerkrachen } & \text { zerkratzen } & \text { zerkriegen } \\ \text { zerkrümeln } & \text { zerlassen } & \text { zerlatschen } & \text { zerlaufen } \\ \text { zerlegen } & \text { zerlesen } & \text { zerliegen } & \text { zerlöchern } \\ \text { zerlösen } & \text { zermahlen } & \text { zermalmen } & \text { zermanschen } \\ \text { zermartern } & \text { zermatschen } & \text { zermürben } & \text { zernagen } \\ \text { zernichten } & \text { zernieren } & \text { zerpfeifen } & \text { zerpflücken } \\ \text { zerpflügen } & \text { zerplatzen } & \text { zerprügeln } & \text { zerpulvern } \\ \text { zerquälen } & \text { zerquatschen } & \text { zerquetschen } & \text { zerraufen } \\ \text { zerreden } & \text { zerreiben } & \text { zerreißen } & \text { zerrinnen } \\ \text { zerrupfen } & \text { zerrütten } & \text { zersäbeln } & \text { zersägen } \\ \text { zerschaben } & \text { zerschellen } & \text { zerscherben } & \text { zerschießen } \\ \text { zerschlagen } & \text { zerschleißen } & \text { zerschlitzen } & \text { zerschmeißen } \\ \text { zerschmelzen } & \text { zerschmettern } & \text { zerschneiden } & \text { zerschnippeln } \\ \text { zerschrammen } & \text { zerschroten } & \text { zerschrunden } & \text { zerschunden } \\ \text { zerschürfen } & \text { zersetzen } & \text { zersieben } & \text { zersiedeln } \\ \text { zersingen } & \text { zersitzen } & \text { zersorgen } & \text { zerspalten } \\ \text { zerspanen } & \text { zerspellen } & \text { zerspleißen } & \text { zersplittern } \\ \text { zerspratzen } & \text { zersprengen } & \text { zerspringen } & \text { zerstampfen } \\ \text { zerstäuben } & \text { zerwühlen } & \text { zerstieben } & \text { zerstören } \\ \text { zerstoßen } & \text { zerstrahlen } & \text { zerstreiten } & \text { zerteppern } \\ \text { zerstrubbeln } & \text { zerstückeln } & \text { zerteilen } & \text { zertrümmern } \\ \text { zertrampeln } & \text { zertrennen } & \text { zertrirkten } \\ \text { zerwalzen } & \text { zerweichen } & \text { zerwerfen } \\ & & \end{array}$




\section{BIBLIOGRAFÍA}

Balzer, B., Barjau, E., Holst, K., La lengua alemana: su complemento preposicional, Ariel, Barcelona. 1990.

Barjau, E., "Observaciones en torno a la estructura y la morfología del complejo verbal alemán”. Filología moderna 68-70, Madrid, 1980, págs. 27-54.

Barz, I., "Determinanten der Wortbildungsaktivität primärer Lexeme". Beiträge zur Erforschung der deutschen Sprache 9, Leipzig, 1989, págs. 32-36,

-, "Graphische Varianten bei der substantisvischen Komposition". Deutsch als Fremdsprache 30, Leipzig, 1993, págs. 167-171.

-, “Zur Wortbildungsaktivität primärer Lexeme”. Beiträge zur Erforschung der deutschen Sprache 7, Leipzig, 1987, págs. 5-11.

Bellmann, G., "Zur Nomination und zur Nominationsforschung". Beiträge zur Erforschung der deutschen Sprache 9, Leipzig, 1989, págs. 28-31.

Beltrán, M., "El verbo en la recodificación de la comunicación no verbal en alemán y en español". Aproximación plural a las lenguas alemana y española. Actas de la VII Semana de Estudios Germánicos: Relaciones hispano-alemanas en la lengua, la literatura y la cultura, celebrada en El Escorial (30 de marzo - 3 de abril de 1992), Madrid, 1995, págs. 75-85.

Castell, A., "Zum Verfahren in vergleichenden Untersuchungen zur Ausklammerung". Revista de filología alemana 1, Madrid, 1993, págs. 123-130.

Cohen, D., El aspecto verbal, Visor libros, Madrid, 1993.

Colbert, B., "Zur Frage der Präfixbildungen”. Deutsch als Fremdsprache 3, Leipzig, 1966, págs. 12-16.

Egg, M., Aktionsart und Kompositionalität, Akademie Verlag, Berlín, 1994.

Fandrych, Ch., Wortart, wortbildungsart und kommunikative Funktion, Tübingen, Niemeyer, 1993.

Fandrych, Ch., Thurmair, M., "Ein Interpretationsmodell für Nominalkomposita: linguistische und didaktische Überlegungen". Deutsch als Fremdsprache 31, Leipzig, 1994, págs. 34-44.

Filiceva, N., "Zur semantischen Isofunktionalität von Wortverbindung und Kompositum im Deutschen". Deutsch als Fremdsprache 29, Leipzig, 1992, págs. 84-89.

Fleischer, W., Barz, I., Wortbildung der deutschen Gegenwartssprache, Tübingen, Niemeyer, 1992.

Gataullin, R., "Zu wortbildenden Textpotenzen”. Deutsch als Fremdsprache 27, Leipzig, 1990, págs. 240-245.

Harnisch, K. R., "Doppelpartikelverben als Gegenstand der Wortbildungslehre und Richtungsadverbien als Präpositionen. Ein syntaktischer Versuch". Tendenzen verbaler Wortbildung in der deutschen Gegenwartssprache, Helmut Buske Verlag, Hamburgo, 1982, págs. 107-134.

Harras, G. (Ed.), Die Ordnung der Wörter. Berlín / New York, 1995. 
Hinderling, R., "Konkurrenz und Opposition in der verbalen Wortbildung". Tendenzen verbaler Wortbildung in der deutschen Gegenwartssprache, Helmut Buske Verlag, Hamburgo, 1982, págs. 81-107.

Kreiser, L., Gottwald, S., Stelzner, W. (Eds.), Nichtklassische Logik, Berlin, Akademie Verlag, 1990.

Kühnhold, I., Wellmann, H., Deutsche Wortbildung, Typen und Tendenzen in der Gegenwartssprache, Schwann, Düsseldorf, 1973.

Kunsmann, P., Verbale Gefüge, Hueber, München, 1973.

Lerot, J., Transformationelle Behandlung der Verbzusammensetzungen im Deutschen, Schwann, Düsseldorf, 1971.

Matussek, M., Wortneubildung im Text, Hamburg, Helmut Buske Verlag., 1994.

Motsch, W., "Wieviel Syntax brauchen Komposita?" Festschrift für Wolfgang Fleischer, Francfort, 1992, págs. 71-81.

Olsen, S., Wortbildung im Deutschen, Kröner, Stuttgart, 1986.

—, "Zur Suffigierung und Präfigierung in verbalem Bereich des Deutschen". Papiere zur Linguistik 42, Tubinga, 1990, págs. 31-48.

Partee, B. H., Meulen, A., Wall, R. E., Mathematical Methods in Linguistics, Dordrecht, Kluwer, 1990.

Rak, Z., "Fachsprachliche Wortbildung - ihre Lehr- und Lernbarkeit". Deutsch als Fremdsprache 29, Leipzig, 1992, págs. 234-238.

Rickheit, M., Wortbildung. Grundlagen einer kognitiven Wortsemantik. Opladen, 1993.

Sarlov, S., Zur semantischen Struktur von Präfixverben, Niemeyer, Tubinga, 1992.

-, Zur Semantik einiger Ver-Verben und ihrer bulgarischen Entsprechungen, Julius Groos Verlag, Heidelberg, 1992.

Schröder, M., "Warum nicht *umweltlich und *Umweltler?". Deutsch als Fremdsprache 30, Leipzig, 1993, págs. 172-175.

Simeckova, A., "Das trennbare Verb im Deutschen - eine Übergangserscheinung?". Zeitschrift für Germanistische Linguistik 19, Berlín, 1991, págs. 226-234.

-, "Das deutsche komplexe Verb unter anwendungsbezogenem Aspekt: Bei Tische halte ich mich unter / mit Künstlern und Studenten nur...". Deutsch als Fremdsprache 32, Leipzig, 1995, págs. 96-102.

Steinitz, R., "Mehrdeutigkeit in der Grammatik auf und an - eine Fallstudie". Fügungspotenzen, Akademie Verlag, Berlín, 1992, págs. 163-188.

Wegener, H., "Pluralregeln und mentale Grammatik". Fügungspotenzen, Akademie Verlag, Berlín, 1992, págs. 225-250.

Wilss, W., "Schematheorie und Wortbildung". Deutsch als Fremdsprache 28, Leipzig, 1992, págs. 230-233.

Zifonun, G., Zur Theorie der Wortbildung am Beispiel deutscher Präfixverben, Hueber, Munich, 1973. 
